\title{
A REMARK ON CHARACTERISTICS
}

\author{
M. H. MARTIN
}

The algebraic function $\psi(\sigma, \theta)$ defined by $\psi^{3}+3 \sigma \psi-3 \theta=0$ is a solution $^{1}$ to Tricomi's equation

$$
\psi_{\theta \sigma}+\sigma \psi_{\theta \theta}=0
$$

If $\psi$ be taken as the stream function for the flow of the Tricomi gas, the stream lines $\psi=$ const. in the $(\theta, \sigma)$-plane (hodograph plane) constitute a one-parameter family of straight lines enveloping the semicubic parabola $9 \theta^{2}=-4 \sigma^{3}$. This semi-cubic parabola is one of the characteristics of Tricomi's equation. Within the region $9 \theta^{2}<-4 \sigma^{3}$ the solution is triple-valued and the solution surface $\psi=\psi(\theta, \sigma)$ consists of three sheets, two of which unite along curves which project onto the characteristic $9 \theta^{2}=-4 \sigma^{3}$ in the $(\theta, \sigma)$-plane. Thus this characteristic serves as the locus of branch points for the algebraic function $\psi(\theta, \sigma)$.

If Tricomi's equation be replaced by a linear equation

$$
L(u)=a_{i j} u_{x_{i} x_{j}}+b_{i} u_{x_{i}}=0
$$

(the repeated indices denoting summation from 1 to $n$ ) for an unknown function $u$ of $n$ independent variables $x_{1}, \cdots, x_{n}$, it is natural to ask the following question.

If a solution to $L(u)=0$ is defined implicitly by

$$
F\left(x_{1}, \cdots, x_{n}, u\right)=0 \text {, }
$$

will the envelope of the one-parameler family of (n-1)-dimensional hypersurfaces $u=$ const. (assuming that the envelope exists) be a characteristic hypersurface?

To begin with we have

$$
F_{x_{i}}+F_{u} u_{x_{i}}=0, F_{x_{i} x_{j}}+F_{u x_{i}} u_{x_{j}}+F_{u x_{j}} u_{x_{i}}+F_{u u} u_{x_{i}} u_{x_{j}}+F_{u} u_{x_{i} x_{j}}=0,
$$

from which we obtain ${ }^{2}$

$$
L(F)+2 \Delta_{1}\left(F_{u}, u\right)+F_{u u} \Delta_{1}(u, u)=0 .
$$

When this equation is multiplied through by $F_{u}^{2}$, we find

Received by the editors May 28, 1951.

${ }^{1}$ For details on the relation of this solution to gas dynamics, see M. H. Martin and W. Thickstun, An example of transonic flow for the Tricomi gas, Proceedings of the Fourth Symposium on Applied Mathematics.

${ }^{2}$ By $\Delta_{1}(u, v)$ we mean Beltrami's first differential operator, i.e., $\Delta_{1}(u, v)=a_{i j} u_{x_{i}} v_{x_{j}}$. 


$$
F_{u}^{2} L(F)-2 F_{u} \Delta_{1}\left(F_{u}, F\right)+F_{u u} \Delta_{1}(F, F)=0 .
$$

We assume that the one-parameter family of $(n-1)$-dimensional hypersurfaces $F\left(x_{1}, \cdots, x_{n}, u\right)=0$ has an envelope

$$
\Phi\left(x_{1}, \cdots, x_{n}\right)=F\left(x_{1}, \cdots, x_{n}, u\left(x_{1}, \cdots, x_{n}\right)\right)=0,
$$

the function $u\left(x_{1}, \cdots, x_{n}\right)$ being defined implicitly by

$$
F_{u}\left(x_{1}, \cdots, x_{n}, u\right)=0 .
$$

At a point of contact of a member of the family with the envelope, not only does $\Phi=0$, but also

$$
F_{x_{i}}=\Phi_{x_{i}}, \quad F_{u}=0,
$$

to imply, provided $F_{u u} \neq 0$,

$$
\Delta_{1}(\Phi, \Phi)=0 .
$$

TheOREM. If $F\left(x_{1}, \cdots, x_{n}, u\right)=0$ defines implicitly $a$ solution to $L(u)=0$, and if the one-parameter family of $(n-1)$-dimensional hypersurfaces $u=$ const. in the space of the independent variables has an envelope upon which $F_{u u} \neq 0$, the envelope is a characteristic hypersurface. ${ }^{3}$

For a multi-valued solution of $L(u)=0$, the locus of points in the space of independent variables at which exactly two of the branches unite must be a characteristic hypersurface.

\section{UNIVERSITY OF MARYLAND}

${ }^{3}$ This theorem is contained in a theorem of Le Roux and Delassus as quoted by J. Hadamard in his book Le problème de Cauchy et les équations aux dérivées partielles linéaires hyperboliques, Paris, 1932, p. 102. [Referee's note.] 